\title{
Efecto del dopado con Si sobre la estructura de defectos en sistemas heteroepitaxiales GaN/AIN/Si(111)
}

\author{
A. M. SÁNCHEZ, S. I. MOLINA, F. J. PACHECO Y R. GARCíA, M. A. SÁNCHEZ-GARCíA ${ }^{1}$, F. J. SÁNCHEZ ${ }^{1}$ Y E. CALLEJA ${ }^{1}$ \\ Departamento de Ciencia de los Materiales e I.M. y Q.I., Universidad de Cádiz, Apdo. 40, 11510 Puerto Real (Cádiz) \\ ${ }^{1}$ Departamento de Ingeniería Electrónica, ETSI Telecomunicación, U.P.M., Ciudad Universitaria s/n, 28040 Madrid
}

\begin{abstract}
En el presente artículo se lleva a cabo el análisis del efecto que el dopado con Si tiene sobre la estructura de defectos en epicapas de GaN crecidas por epitaxia de haces moleculares sobre sustratos de Si (111) utilizando capas amortiguadoras de AlN. La caracterización estructural se llevó a cabo mediante microscopía electrónica de transmisión convencional y de alta resolución. El dopado con Si afecta a la desorientación y tamaño de los subgranos que constituyen la estructura mosaico de la epicapa de GaN. El dopado con Si provoca un aumento en la densidad de defectos planares, así como una disminución en la densidad de dislocaciones de propagación. El incremento en el grado de desorientación de inclinación, así como en la densidad de defectos planares que se produce conforme aumenta el dopado con Si explican la disminución en la densidad de dislocaciones que alcanzan la superficie libre de GaN.
\end{abstract}

\section{Palabras clave: Nitruro de Galio, GaN/AlN/Si(111), Heteroestructura semiconductora, TEM, HREM}

\section{Si doping effect on the defect structure in GaN/AlN/Si(111) heteroepitaxial systems}

The Si doping effect on the defect structure in GaN epilayers grown by molecular beam epitaxy on $\mathrm{Si}(111)$ substrates using AlN buffer layers has been studied. Transmission electron microscopy and related techniques have been used to carry out the structural characterization. The Si doping affects both the subgrain size and misorientation in GaN epilayer mosaic structure. The Si doping also leads to an increase of the planar defect density, as well as a decrease of the threading dislocation density. The enlargement of the subgrain tilt and the planar defect density explain the reduction of the dislocation density reaching the GaN free surface.

Key words: Gallium nitride, GaN/AlN/Si(111), semiconductor heterostructure, TEM, HREM

\section{INTRODUCCIÓN}

El gran interés que han despertado los semiconductores de banda prohibida ancha en la actualidad se centra en el diseño y elaboración de dispositivos optoelectrónicos de aplicación en la tecnología del almacenamiento de datos, así como de componentes electrónicos de alta potencia y elevada temperatura. La fabricación de diodos con emisión dentro del intervalo de energías que abarca desde $3.4 \mathrm{eV}$ hasta $6.2 \mathrm{eV}{ }^{(1)}$, así como la obtención de diodos láser, han mostrado el enorme potencial de las aleaciones III-N para la producción de componentes electrónicos que operen en el intervalo del espectro visible y U.V. Así, los dispositivos basados en materiales semiconductores III-N son capaces de mejorar la potencia y temperatura de operación debido al elevado valor de las bandas prohibidas que presentan.

Para el crecimiento de GaN se han utilizado una gran variedad de sustratos, entre los que destacan 6H-SiC, GaAs, $\mathrm{MgAl}_{2} \mathrm{O}_{4}$, zafiro y $\mathrm{Si}$, así como diversas técnicas de crecimiento basadas principalmente en deposición de vapores químicos (CVD) y epitaxia de haces moleculares (MBE). El crecimiento de GaN sobre sustratos de $\mathrm{Si}$, tiene particular interés, ya que permitiría su integración en la tecnología del Si, que ha alcanzado un alto grado de desarrollo. Sin embargo, debido al elevado desajuste reticular entre el GaN y el Si (16.9\% en tensión) y a la diferencia en los coeficientes de expansión térmica $\left(\alpha_{\mathrm{GaN}}=5.59 / \mathrm{K}\right.$ y $\left.\alpha_{\mathrm{Si}}=3.59 / \mathrm{K}\right)$, aparecen dificultades en el crecimiento epitaxial. Por ello, se hace necesaria la utilización de capas amortiguadoras que mejoren la calidad cristalina de la posterior epitaxia de $\mathrm{GaN}^{(2)}$. Por otra parte en el sistema que nos ocupa resulta fundamental el uso de capas amortiguadoras debido a que en el crecimiento de GaN directamente sobre Si se produce la formación de una capa amorfa en la intercara ${ }^{(3,4)}$, dificultando el crecimiento de epitaxias de nitruros con calidad suficiente para la fabricación posterior de dispositivos.

En el presente trabajo se analiza la influencia del dopado con Si sobre la calidad estructural de heteroepitaxias GaN/AlN/ $\mathrm{Si}(111)$ mediante microscopía electrónica de transmisión y se estudian los mecanismos responsables de los cambios estructurales que tienen lugar en tales sistemas.

\section{PROCEDIMIENTO EXPERIMENTAL}

El estudio se realizó a partir de una serie de películas de GaN dopadas con distintas concentraciones de $\mathrm{Si}$, crecidas mediante epitaxia de haces moleculares (MBE) sobre sustratos de Si(111) tipo-p, usando una capa amortiguadora optimizada de $\operatorname{AlN}^{(5,6,7)}$. La temperatura de crecimiento de la capa de $\mathrm{GaN}$ fue de $760^{\circ} \mathrm{C}$ y el espesor de capa crecida fue $0.8 \mu \mathrm{m}$. En el presente artículo se estudian tres muestras: (i) $\mathrm{GaN} / \mathrm{AlN} / \mathrm{Si}(111) \sin$ dopar, que denominaremos $\mathrm{D}_{\mathrm{o}}$ (ii) Heteroestructura $\mathrm{D}_{1}, \mathrm{GaN} / \mathrm{AlN} / \mathrm{Si}(111)$ 
dopada con una concentración de portadores negativos $\mathrm{N}_{\mathrm{Si}}=$ 1.1.10 $\mathrm{cm}^{-3}$ y (iii) Heteroestructura $\mathrm{D}_{2}$, GaN/AlN/Si(111) con una concentración $\mathrm{N}_{\mathrm{Si}}=6.0 .10^{17} \mathrm{~cm}^{-3}$.

La preparación de las muestras electrón-transparentes se realizó mediante adelgazamiento mecánico y posterior bombardeo iónico con iones $\mathrm{Ar}^{+}$. La caracterización de las muestras se realizó mediante Difracción Electrónica de Área Seleccionada (SAED)y Microscopía Electrónica deTransmisión Convencional (TEM), tanto en sección transversal (XTEM) como en visión planar (PVTEM) en un microscopio Jeol 1200 EX operando a $120 \mathrm{kV}$. También se han realizado estudios de Microscopía Electrónica de Transmisión de Alta Resolución (HREM) en un microscopio Jeol 2000 EX operando a 200kV.

\section{RESULTADOS Y DISCUSIÓN}

Los estudios mediante SAED de muestras preparadas en sección transversal de las distintas epicapas muestran que las epicapas de GaN presentan una estructura hexagonal tipo wurtzita, con la siguiente relación de orientación con el sustrato de Si sobre el que se ha realizado el crecimiento:

$$
\text { [11르] GaN // [110] Si y [0001] GaN / / [111] Si }
$$

A partir de la elongación de los puntos de los diagramas SAED de amplias zonas de las muestras en visión planar como el que se presenta en la figura 1, se deduce que las capas de GaN presentan estructura mosaico constituida por subgranos ligeramente desorientados. Dicha estructura es normal que aparezca debido al elevado desajuste reticular existente entre el GaN y el sustrato de $\mathrm{Si}^{(8)}$. Este desajuste da lugar a la formación de una distribución no homogénea de dislocaciones en la intercara con el sustrato, lo cual provoca que los subgranos constituyentes de la epicapa crezcan desorientados. Hemos de considerar dos tipos de desorientaciones posibles, las cuales se esquematizan en la figura 2. Una desorientación de rotación alrededor del eje [0001] y una desorientación de inclinación en torno a ejes que están contenidos en el plano de crecimiento (0001), dando lugar a la formación de dislocaciones en la frontera de dichos granos. Debido a la desorientación de inclinación, en los límites de grano aparecen dislocaciones de arista cuya línea de dislocación es paralela al plano de crecimiento (0001) (ver figura 2a). En el caso de la rotación de granos respecto a un eje paralelo a la dirección [0001], se produce la formación de dislocaciones de arista con la línea de dislocación perpendicular al plano de crecimiento (0001) (ver figura 2b).

En definitiva, las epicapas de GaN están constituidas por granos columnares que alcanzan la superficie libre de la epicapa crecida. A partir de series de imágenes de PVTEM de las distintas muestras se llevó a cabo un estudio de la distribución de tamaños de grano con ayuda del sistema descrito en la referencia 9. Se observó una disminución del tamaño medio de los subgranos conforme aumentaba la concentración de portadores negativos en la epicapa, como se recoge en la Tabla I. Por otro lado, el factor de forma de dichos subgranos resultó ser de

TABLA I. TAMAÑO MEDIO DE LOS SUBGRANOS QUE CONSTITUYEN LA ESTRUCTURA MOSAICO DE LAS EPICAPAS DE GAN

\begin{tabular}{|lccc|}
\hline Muestra & $\mathrm{D}_{0}$ & $\mathrm{D}_{1}$ & $\mathrm{D}_{2}$ \\
\hline Tamaño medio de los subgranos $(\mathrm{nm})$ & 280 & 205 & 175 \\
\hline
\end{tabular}

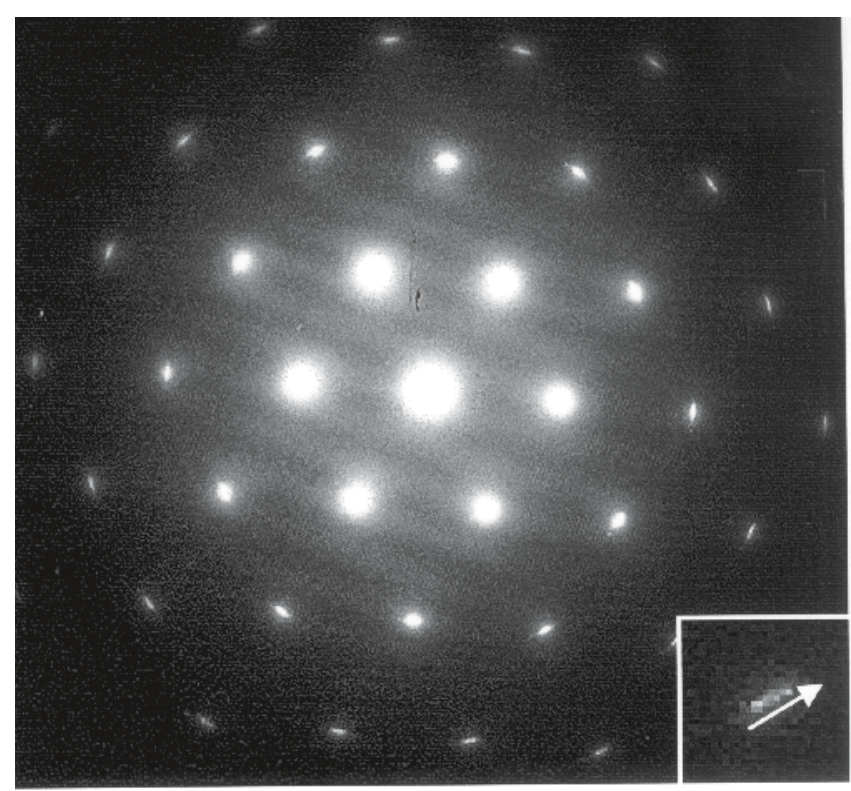

Figura 1. Diagrama de difracción de área seleccionada en el eje $<0001>$. La reflexión (0220) aparece ampliada en la esquina superior derecha del diagrama.

a
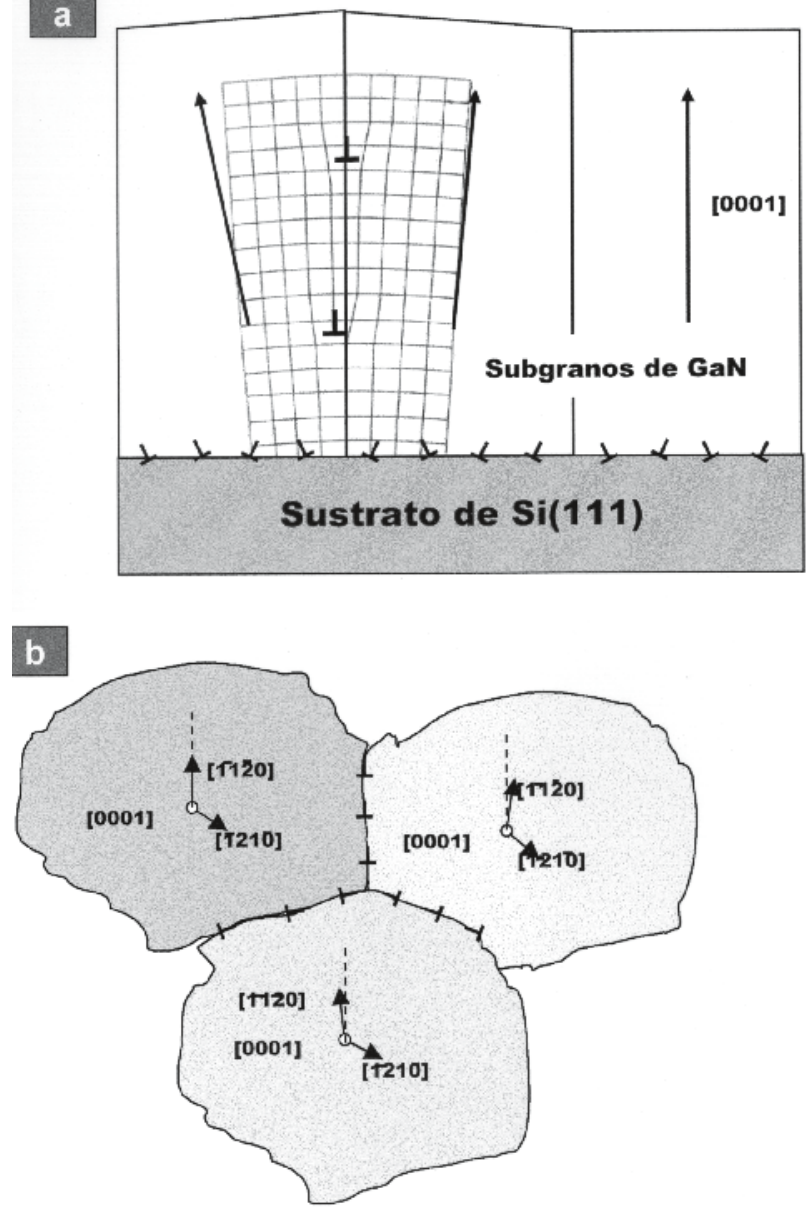

Figura 2. Representación esquemática de la desorientación en los subgranos que constituyen la estructura mosaico (a) desorientación de inclinación y (b) desorientación de rotación. 
0.6 en todos los casos. Es decir, no se ve afectado de forma apreciable por el nivel de dopado de la epicapa.

Mediante el análisis de diagramas SAED en muestras en visión planar de subgranos adyacentes, se estimó la desorientación de inclinación existente entre dichos granos en las distintas muestras. De este modo, se comprobó que la desorientación de inclinación de los subgranos era de $2.0^{\circ} \pm 0.5^{\circ}$ para las muestras dopadas con $\mathrm{Si}$, valor mayor que el medido para la muestra no dopada, $1.0^{\circ} \pm 0.5^{\circ}$.

En diagramas SAED, como el de la figura 3, que corresponde a zonas de las muestras de $65 \mathrm{~mm}^{2}$, se observaron puntos de difracción extra rotados $30^{\circ}$ con respecto a los principales, de mayor intensidad, etiquetados con la letra R. Este hecho se debe a la existencia de granos rotados $30^{\circ}$ alrededor del eje [0001] con respecto a la matriz que los circunda. La existencia de estos granos también ha podido comprobarse mediante HREM donde puede medirse fácilmente la desorientación entre uno de estos granos rotados $30^{\circ}$ y el resto del material, como se indica en la figura 3(b). En esta figura se han trazado un par de vectores en una misma dirección en dos granos contiguos para dejar constancia de la rotación de $30^{\circ}$ entre ellos.
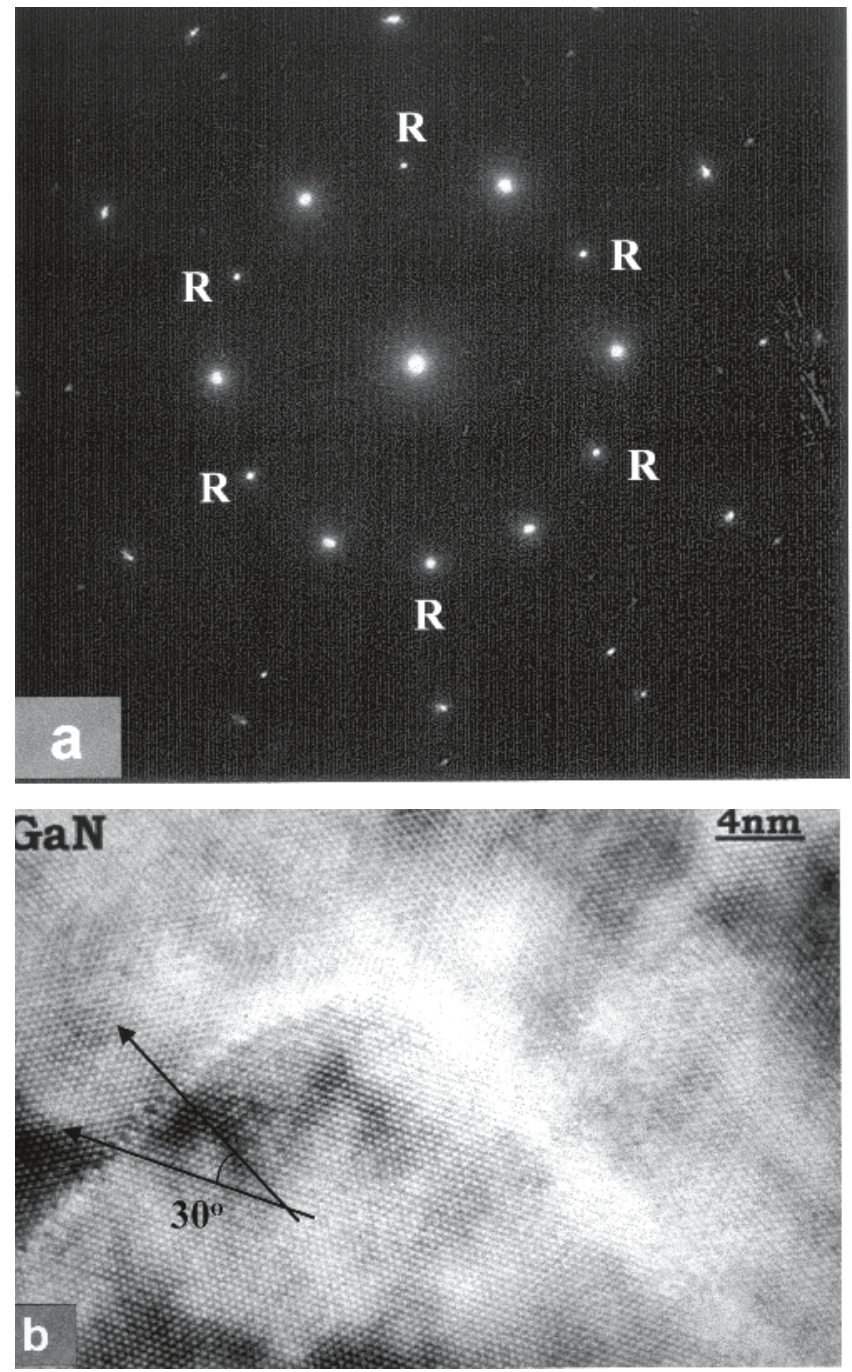

Figura 3. (a) Diagrama de difracción de área seleccionada en el eje $<0001>$ mostrando puntos extras rotados $30^{\circ}$ etiquetados con la letra R y (b) Imagen de HREM de una frontera de grano entre un grano rotado $30^{\circ} \mathrm{y}$ otro adyacente.
En todas las muestras se han observado estos granos rotados $30^{\circ}$ con respecto a la matriz hexagonal, pero su densidad es mayor en las muestras dopadas.

Un parámetro muy importante de la calidad estructural de la epicapa de GaN es la densidad de dislocaciones. Para determinar las densidades de dislocaciones de propagación que alcanzan la superficie libre de la muestra, se llevó a cabo el análisis de imágenes de PVTEM de amplias zonas de las muestras que contienen cientos de granos. Para este estudio se tomaron imágenes en condiciones de haces débiles como la presentada en la figura 4 , y se aplicó el criterio de invisibilidad convencional, g.b=0. El valor de la reflexión g utilizada fue $\mathrm{g}=1120$, próximo al polo $\langle 0001>$, con la que son visibles únicamente las dislocaciones cuyos vectores de Burgers son del tipo

$$
\mathrm{b}=1 / 3\langle 11 \overline{2} 0\rangle \quad \mathrm{y} \mathrm{b}=1 / 3\langle 1 \overline{12} 3\rangle
$$

Bajo estas condiciones no es posible observar dislocaciones de tornillo puras. No obstante, la densidad de estas ultimas se reduce enormemente durante el crecimiento de la película de GaN debido a la formación de semilazos ${ }^{(10)}$. Los valores estimados de las densidades de dislocaciones de propagación que alcanzan las superficies libres de las capas de GaN quedan recogidas en la Tabla II.

Por otra parte, en las epicapas de GaN están presentes otros defectos estructurales: (i) dominios de inversión que se propagan verticalmente a lo largo de la dirección [0001] como se observa en la figura 5(a) y (ii) defectos planares en el plano de crecimiento (0001), figura 5(b). Los dominios de inversión se

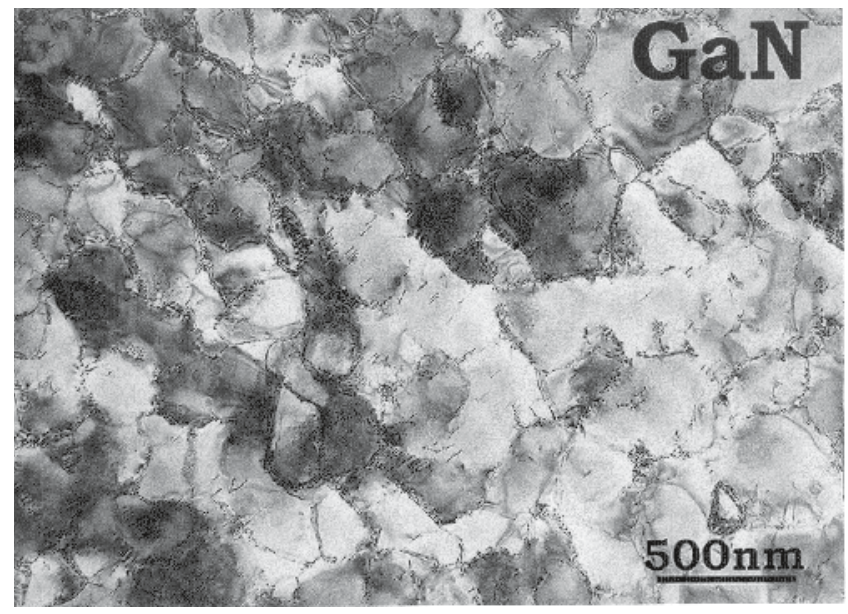

Figura 4. Micrografía TEM en visión planar en condiciones de dos haces con $\mathrm{g}=1120$ de la capa de GaN.
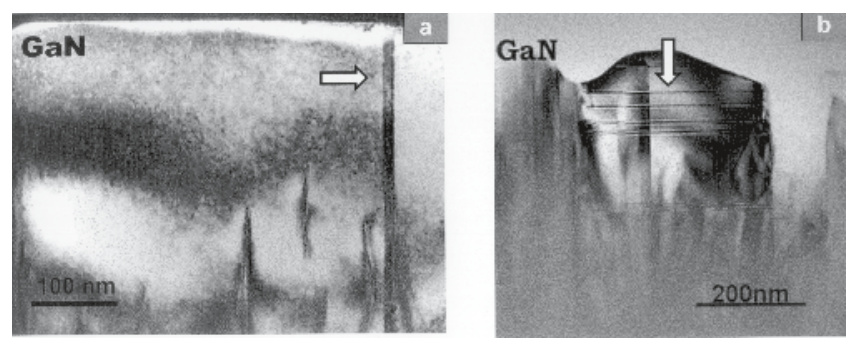

Figura 5. (a) Imagen de XTEM en condición de dos haces con $\mathrm{g}=0002$ de un dominio de inversión y (b) imagen XTEM en condiciones de dos haces con $\mathrm{g}=1 \overline{1} 00$, evidenciando la existencia de defectos planares. 
TABLA II. DENSIDAD DE DISLOCACIONES PARA MUESTRAS CON DIFERENTE CONCENTRACIÓN DE PORTADORES NEGATIVOS (NSI).

\begin{tabular}{|lccc|}
\hline Muestra & $\mathrm{D}_{0}$ & $\mathrm{D}_{1}$ & $\mathrm{D}_{2}$ \\
\hline Densidad de dislocaciones $\left(10^{9} \mathrm{~cm}^{-2}\right)$ & $6.4 \pm 0.5$ & $5.3 \pm 0.5$ & $0.8 \pm 0.2$ \\
\hline
\end{tabular}

determinaron siguiendo el procedimiento descrito por L. T. Romano et al. ${ }^{(11)}$. Para ello se tomaron varias imágenes en condiciones de campo oscuro múltiple con $\mathrm{g}= \pm 0002$ a lo largo de un eje no centrosimétrico, de forma que los dominios de inversión aparecen con contraste invertido al cambiar las condiciones. Además para confirmar la existencia de tales dominios invertidos, se analizaron imágenes adquiridas en condiciones de dos haces con $g=1 \overline{1} 00$ cerca del eje de zona $<1 \overline{1} 20>$, de forma que dichos dominios no aparecen como contrastes verticales en la imagen.

Mediante análisis por contraste de difracción de los defectos planares de la epicapa de GaN, se concluye que dichos defectos son fallos de aplilamiento producidos en el plano basal de crecimiento. Se ha observado que la densidad de defectos planares en la epicapa crece conforme aumenta el contenido de dopante en la misma. Estos fallos de apilamiento también han sido observados mediante HREM, y usualmente se encuentran apilados a lo largo de la dirección de crecimiento [0001] separados distancias del orden de decenas de nanómetros.

Como hemos visto anteriormente, la densidad de dislocaciones que alcanzan la superficie libre de GaN disminuye conforme aumenta el contenido de Si en la epicapa. Por otra parte, conforme aumenta $\mathrm{N}_{\mathrm{Si}}$ se produce un incremento de la densidad de defectos planares, así como un mayor grado de desorientación de inclinación de los subgranos que constituyen la estructura mosaico de la película.

Se ha observado mediante TEM que estos defectos planares localizados en los planos (0001), interaccionan con las dislocaciones de propagación, de forma que contribuyen a disminuir el número de dislocaciones verticales que alcanzan la superficie. Este tipo de interacciones había sido observado previamente en crecimientos de capas de nitruros crecidas sobre zafiro $^{(12)}$. Otro factor que podría contribuir a la disminución de dislocaciones con el dopado de Si es el aumento de dislocaciones horizontales asociadas a la desorientación de inclinación entre subgranos adyacentes, que disminuirían las dislocaciones propagadas verticalmente por eventuales interacciones con éstas.

\section{CONCLUSIONES}

El dopado con Si de heteroestructuras en las que se ha crecido $\mathrm{GaN}$ por MBE sobre sustratos de $\mathrm{Si}(111)$, utilizando una capa amortiguadora de AlN, es una buena aproximación para reducir la densidad de dislocaciones de propagación con vectores de Burgers

$$
\mathrm{b}=1 / 3\langle 11 \overline{2} 0\rangle \quad \mathrm{y} b=1 / 3\langle 11 \overline{2} 3\rangle
$$

que alcanzan la superficie libre de GaN. La dependencia de la desorientación de los subgranos que constituyen la estructura mosaico y de la densidad de defectos planares con la concentración de portadores negativos es fundamental para explicar la mejora de la calidad de las epicapas de GaN dopadas con Si. Como consecuencia, se produce una disminución en un orden de magnitud en la densidad de dislocaciones de propagación mediante el dopado con Si.

\section{AGRADECIMIENTOS}

Este trabajo ha sido financiado por el M.E.C. a través de la CICYT mediante el proyecto No. MAT98-0823-C03-02 y por la Junta de Andalucía a través del grupo de investigación, PAI TEP 0120.

\section{BIBLIOGRAFIA}

1. S. Nakamura y G. Fasol, “The Blue Laser Diode”. Springer (1997)

2. W. J. Meng, J. A. Sell, T. A. Perry, L. E. Rehn y P. M. Baldo, “Growth of aluminium nitride thin films on $\mathrm{Si}(111)$ and $\mathrm{Si}(001)$ : Structural characteristics and development of intrinsic stresses", J. Appl. Phys., 75, 3446-3455 (1994).

3. A. Ohtani, K. S. Stevens y R. Beresford, "Microstructure and photoluminescence of GaN grown on $\mathrm{Si}(111)$ by plasma-assisted molecular beam epitaxy", Appl. Phys. Lett., 65, 61-63 (1994).

4. A. Bourret, A. Barski, J. L. Rouvière, G. Renaud y A. Barbier, "Growth of aluminium nitride on (111) silicon: Microstructure and interface structure", J. Appl. Phys., 83, 2003-2009 (1998).

5. M. A. Sánchez-García , E. Calleja, F. J. Sánchez, F. Calle, E. Monroy, D. Basak, E. Muñoz, C. Villar, A. Sanz-Hervás, M. Aguilar, J. J. Serrano y J. A. Blanco "Growth optimization and doping with $\mathrm{Si}$ and Be of high quality GaN on $\mathrm{Si}(111)$ by molecualr beam epitaxy", J. Electron. Mater., 27, 276-281 (1998).

6. E. Calleja, M. A. Sánchez-García, E. Monroy, F. J. Sánchez, E. Miuñoz, A. SanzHervás, C. Villar y M. Aguilar, "Growth kinetics and morphology of high quality AlN grown on $\mathrm{Si}(111)$ by plasma-assisted molecular beam epitaxy", J. Appl. Phys., 82, 4681-4683 (1997).

7. M. A. Sánchez-García, E. Calleja, E. Monroy, F. J. Sánchez, F. Calle, E. Muñoz, R. Beresford, "The effect of the III/V ratio and substrate temperature on the morphology and properties of GaN- and AlN-layers grown by molecular beam epitaxy on Si(111)", J. Crys. Growth, 183, 23-30 (1998).

8. V. Srikant, J. S. Speck, y D. R. Clarke, "Mosaic structure in epitaxial thin films having large lattice mismatch", J. Appl. Phys. 82, 4286-4295 (1997).

9. S. I. Molina, A. M. Sánchez, F. J. Pacheco, R. García, M. A. Sánchez-García y E. Calleja, "The role of AlN Buffer layers to improve the growth of Si doped GaN on Si(111)", Laticce-Mismatched Thin Film, E.A.Fitzgerald, ed., Warrendale, PA(USA), 177-182 (1999).

10. J. L. Rouvière, M. Arlery, B. Daudin, G. Feuillet y O. Briot, "Transmission electron microscopy structural characterisation of GaN layers grown on (0001) sapphire", Mat. Sci. Eng. B 50, 61-71 (1997).

11. L. T. Romano, J. E. Northup y M. A. O’Keefe, "Inversion domains in GaN grown on sapphire", Appl. Phys. Lett., 69, 2394-2396 (1996).

12. Z. Liliental-Weber, S. Ruminov, Ch. Kisielowski, Y. Chen, W. Swider, J. Washburn, N. Newman, A. Gassmann, X. Liu, L. Schloss, E. R. Weber, I. Grzegory, M. Backowski, J. Jun, T. Suski, K. Pakula, J. Baranowski, S. Porowski, H. Amano, I. Akasaki, "Structural defects in heteroepitaxial and homoepitaxial GaN", Mat. Res. Soc. 395, 351-362 (1996). 